\title{
Effect of splat-interface discontinuity on effective thermal conductivity of plasma sprayed thermal barrier coating
}

\author{
Shi-yi Qiu ${ }^{\mathrm{a}, \mathrm{b}}$, Yan-Chi Liu ${ }^{\mathrm{b}}$, Hong-bo Guo ${ }^{\mathrm{a}}$, Chen-Guang Huang ${ }^{\mathrm{b}}$, Yue Ma ${ }^{\mathrm{a}, * *}$, Chen-Wu Wu ${ }^{\mathrm{b}, *}$ \\ ${ }^{a}$ School of Materials Science and Engineering, Beijing Key Laboratory for Advanced Functional Material and Thin Film Technology, Beihang University (BUAA), Beijing, \\ 100191, PR China \\ ${ }^{\mathrm{b}}$ Institute of Mechanics, Chinese Academy of Sciences, Beijing, 100190, China
}

\section{A R T I C L E I N F O}

\section{Keywords:}

Thermal barrier coatings

Plasma spraying

Interface discontinuity

Thermal conductivity

\begin{abstract}
A B S T R A C T
The thermal barrier coating obtained by atmospheric plasma spraying (APS TBCs) has a distinct lamellar microstructure, in which the splats discontinuous interfaces running parallel to the metal/ceramic interface contribute largely to the reduction in the effective thermal conductivity of APS TBCs. The dependency of such contribution on the topological structure of the interface discontinuity is investigated in the present work. Firstly, the concept of discontinuity of splats interfaces was defined to quantify the splats discontinuous interfaces revealed by microscopic observations. Then, the microstructure model with a random distribution of discontinuous interfaces was established by utilizing the finite element simulation method to investigate the effect of interlayer discontinuity on thermal conductivity of the APS TBCs. Finally, an optimal topological structure of the interface discontinuity was found to be responsible for the lowest effective thermal conductivity of the APS TBCs and typical parametrical tendencies demonstrated.
\end{abstract}

\section{Introduction}

Thermal barrier coatings (TBCs) are applied to protect substrate metal from high-temperature gas and lower metal temperature, consisting of a ceramic top coat and a bond layer. The top coat, usually a kind of oxide ceramics, is primarily used to resist the heat flux, and the bond layer is applied to improve the adhesion of the ceramic top coat [1-3]. By utilizing different process, different microstructures of the ceramic coat could be realized to optimize the overall performance of the TBCs. Air plasma spray (APS), a low-cost method for depositing ceramic thermal barrier coatings (TBCs), can produce the lamellar structure ceramic TBCs, which have the ubiquitous 'splat' boundaries/ interfaces in APS TBCs. These 'splat' boundaries run parallel to the metal/ceramic interface and are thought to be highly effective in reducing the thermal conductivity of APS TBCs [4,5] as the interface discontinuity would always increase the thermal resistance therein.

As a matter of fact, the effective thermal conductivity of the APS TBCs is affected by such microstructures as interfaces, pores, cracks, grain size and so on. Therefore, many researchers have taken various experimental methods to explore the effects of microstructure in TBCs on its thermal conductivity. For instances, Chi [6] and Markocsan [7] et al. regulated the microstructure of the coating by changing the spraying parameters and using different powders to study the thermal properties of YSZ coatings with different microstructure. The results confirm the vital role of interlamellar pores and splat interfaces on the effective thermal conductivity of the coating and enable opportunities for microstructural tailoring through proper selection of feedstock material and process conditions. Nitin P. Padture et al. used the solution precursor plasma spray (SPPS) process to deposit YSZ TBCs with engineered microstructures, claiming the layered-SPPS coating that contains alternate layers of low and high porosities could get excellent thermal insulation properties [8]. Guo combined low thermal conductivity materials $\left(\mathrm{LaPO}_{4}\right.$ and $\left.\mathrm{GdPO}_{4}\right)$ with yttria-stabilized zirconia (YSZ), and utilized air plasma spay to fabricate the nanostructure $\mathrm{LaPO}_{4} / \mathrm{YSZ}$ and GdPO4/YSZ double-ceramic-layer thermal barrier coatings, which are of ultralow thermal conductivity $[9,10]$. In addition, some researchers have also studied the effect of microscopic crystal structure of the coatings on its effective thermal conductivity. Wang [11,12] and Feng [13] investigated the relationships among the crystal structure, phase composition and thermophysical properties of

\footnotetext{
* Corresponding author. No.15 Beisihuanxi Road, Beijing, 100190, China.

** Corresponding author. No. 37 Xueyuan Road, Beijing, 100190, China.

E-mail addresses: mayue@buaa.edu.cn (Y. Ma), chenwuwu@imech.ac.cn, c.w.wu@outlook.com (C.-W. Wu).
} 
materials by $\mathrm{TiO}_{2}$ doped $\mathrm{YSZ}$ and $\mathrm{Al}_{2} \mathrm{O}_{3}$ doped $\mathrm{Gd}_{3} \mathrm{TaO}_{7}$, respectively. At the same time, many researchers have also used some theoretical methods to study the thermophysical properties of thermal barrier coatings. For example, Jin studied the relationship between the effective thermal conductivity and the microstructure in the plasma sprayed yttria-stabilized zirconia (YSZ) coatings based on the Lattice Boltzmann method (LBM) and the effective thermal conductivity of TBCs fabricated by different plasma spray technologies are estimated [14]. Wei et al. proposed an analytical mathematical formulation to indicate the relationship between defects and effective thermal conductivity and the effects of shape and size of defects on the effective thermal conductivity of TBCs are presented [15]. Sevostianov and Kachanov established a model to calculate the anisotropic effective thermal conductivity of the plasma sprayed ceramic coatings in terms of the relevant microstructural parameters, wherein the dominant porous space was treated as the oblate(crack-like) pores [16]. T. Nakamura et al. [17] and Y. Wang et al. [18] set up a detailed finite element model based on the actual porous structure of the coating to investigate the effects of pore sizes, shapes and orientations on the mechanical properties of TBCs. Kulkarni and Allen quantified microstructure (porosity, opening dimensions, orientation and morphologies) in plasma sprayed YSZ thermal barrier coatings through small-angle neutron scattering (SANS) data, and assembled these microstructural parameters into finite element model to predict thermal conductivity and elastic modulus of the coatings [19].

However, in the aforementioned theoretical study of the influence of coating microstructure on thermal conductivity, the researchers focused on the impact of pores on thermal conductivity and studied the size, shape and distribution of pores respectively. As a result, there was often an obvious deviation between the theoretical results and the experimental results, which could be due to the fact that the effect of the discontinuous interface between layers on the thermal conductivity was overlooked. Some scholars began to pay attention to this issue and had characterized the splat interfaces in the plasma spraying coatings. Wang [20] et al. observed the formation process of the splat interface, and stated that not all of the flattened areas of molten droplets were bonded perfectly. There're lots of discontinuous interfaces between splats, so the thermal conductivity in the discontinuous splat interface area would decrease obviously. By comparing the thermal conductivity of the spray coating and the thermal cycle coating, they demonstrated the significant impact of the splat interface on performances of the TBCs. Moreover, Golosnoy et al. analyzed the heat flow across the splat interface and claimed that the effective thermal conductivity is largely controlled by the inter-splat bridge area based on a one-dimensional analytical mode [21], in which the heat flow along the paths paralleling to the interfaces have not been taken into account. Lu et al. characterized the porous structure of the plasma sprayed coating and revealed numerically that the splat interface has a significant effect on the effective thermal conductivity [22]. However, there is no quantitative description on the effect of the discontinuous splat interface on the effective thermal conductivity of APS TBCs, in particular the influence of topology of the interface discontinuity. This is a critical factor for optimize the thermal performance of such APS TBCs through designing the dimensions and locations of the interface discontinuities.

The dependency of the effective thermal conductivity of the APS TBCs on the topology of the interface discontinuity is concentrated on this paper. Firstly, the discontinuity of splat interfaces is quantified and the location of the discontinuous interfaces is mathematically described. Then, the finite element model is used to simulate the heat flow through the TBCs and calculate the effective thermal conductivity, which is verified by the experimental data. Finally, the effect of splat interfaces discontinuity on thermal conductivity is investigated, and the optimized topology of the discontinuous interface is obtained under the present conditions. These methods as well results could be utilized for the microstructure design of APS TBCs.
Table 1

Spray parameters used for sprayed YSZ top coatings.

\begin{tabular}{lllllll}
\hline $\mathrm{Ar}$ & $\mathrm{He}$ & $\mathrm{U}$ & $\mathrm{I}$ & $\mathrm{D}$ & $\mathrm{F}$ & $\mathrm{G}$ \\
\hline$(\mathrm{L} / \mathrm{min})$ & $(\mathrm{L} / \mathrm{min})$ & $(\mathrm{V})$ & $(\mathrm{A})$ & $(\mathrm{mm})$ & $(\mathrm{g} / \mathrm{min})$ & $(\mathrm{Mpa})$ \\
27 & 1.67 & 75 & 600 & 85 & 21 & 0.4
\end{tabular}

Ar/He: Flow of Ar/He; U: Voltage; I: Electric current; D: Spray distance; F: Powder feed rate; G: Cooling gas pressure.

\section{Experiments and modeling}

\subsection{Coating preparation and microstructures analysis}

The YSZ coatings were prepared by ZB-80X plasma spraying equipment with $\mathrm{YSZ}\left(8 \mathrm{wt} \% \mathrm{Y}_{2} \mathrm{O}_{3}\right.$ ) powders. The stainless steel wafer of diameter $12.7 \mathrm{~mm}$ was used as a substrate to be sprayed, after roughened by sandblasting. The spraying parameters are showed in Table 1. Since the thermal expansion coefficients of the substrate and the coating are different, the substrate and the coating could be separated by heating the substrate and then cooling in water to obtain a separate coating sample. The thickness of the separate coating is $1.375 \mathrm{~mm}$. The density of the sample was determined by the Archimedes method, which is $5.1386 \mathrm{~g} / \mathrm{cm}^{3}$. The porosity of the coating samples was quantified by the method of image analysis. Firstly, scanning electron microscopy (SEM) was adopted to observe and screen the microstructure upon the polished cross-sections of the coatings, and then the cross-sectional images of the coating are treated by threshold method to count the pores within the coating. The pores can be characterized by two types of pores, including the pores in every single layer and those at the discontinuous interfaces. Finally, the commercial software Image $\mathrm{J}$ was utilized to calculate the porosity only containing the volume fraction of the pores within every single layer, which is averagely $8.08 \%$ for the coating samples in the present work.

The cross sections of the sample were observed by Quanta $200 \mathrm{~F}$ field emission environment scanning electron microscope. The typical SEM micrographs of APS coatings specimens are shown in Fig. 1. It is found that the 8YSZ coating sample coated by APS is a typical layered structure, and the stacking of layers was observed upon the fracture surfaces. The thickness of each layer of the batch is roughly $10.25 \mu \mathrm{m}$ by statistics and the statistical characteristics of the thickness of each layer is shown in Table 2 . The interface discontinuities can be clearly found between the adjacent layers and every layer is filled with pores or separated regions. These features have been largely considered in the present simulation studies.

\subsection{Thermal conductivity measurement}

The thermal conductivity of the coating has been obtained indirectly. Firstly, the thermal diffusivity of the coating was gained by the method of laser flash [23], and then the specific heat capacity and density of the coating were measured, and finally the thermal conductivity $\kappa$ was calculated by the formula:

$\kappa=\phi \times C_{p} \times \rho$

During the measurement of the thermal diffusivity of the coating with the instrument FLA427, the forward side of the sample was irradiated by the pulsed Laser, and the temperature at the backward side of the sample was monitored and recorded. And then the time when the temperature of backward side of the sample reached half of its maximum temperature was adopted to estimate the thermal diffusivity of the sample as [23]:

$\alpha=0.1388 \frac{L^{2}}{t_{1 / 2}}$ 

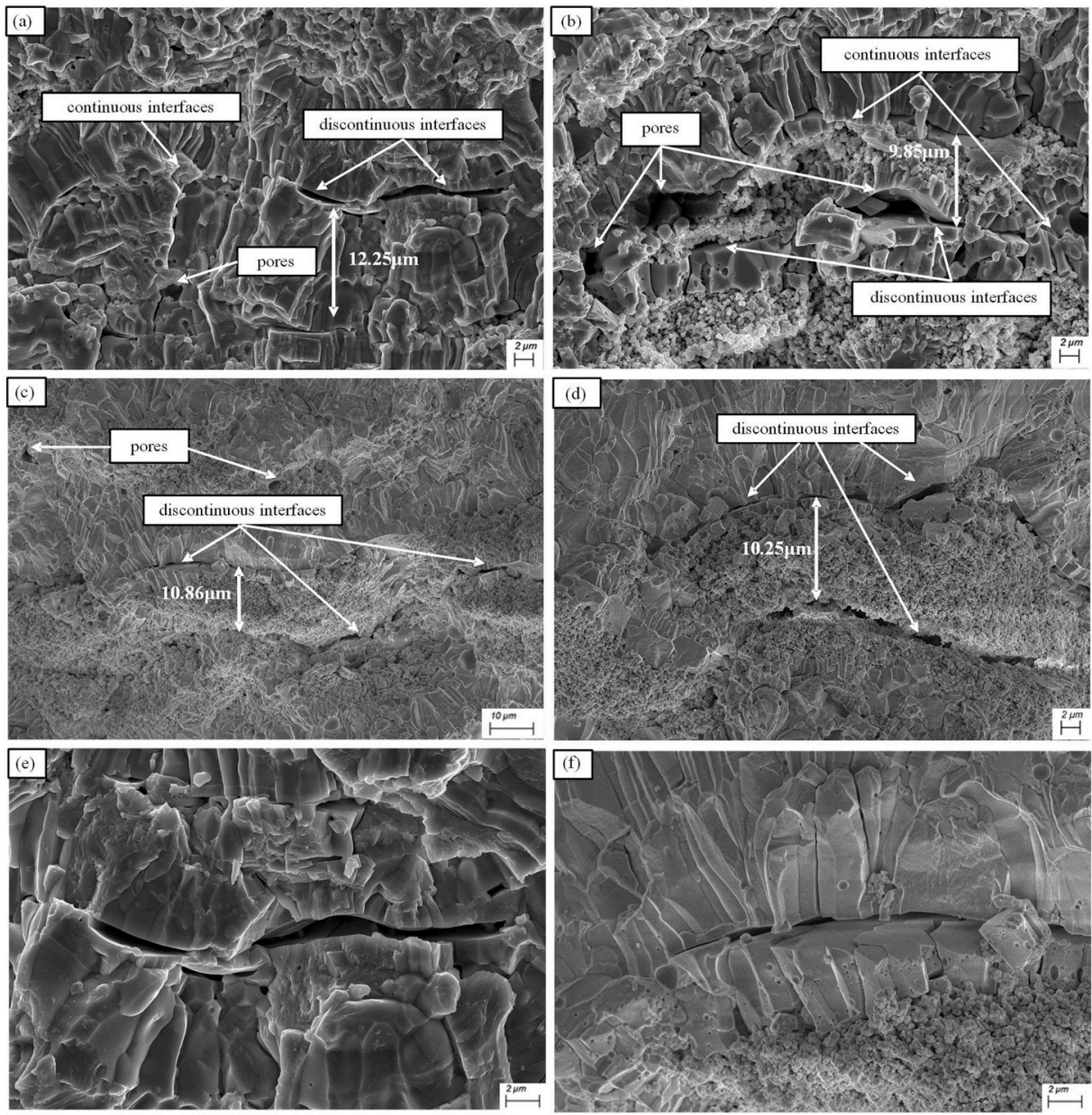

Fig. 1. (a), (b), (c), (d) SEM micrograph of the APS coatings, (e) and (f). SEM micrograph of discontinuous interfaces.

The specific heat capacity of $8 \mathrm{YSZ}, \mathrm{C}_{\mathrm{p}}$ is a function of temperature and can be calculated from the content of the oxide components and their respective standard specific heat capacities. The respective standard specific heat capacity of each temperature of $\mathrm{Y}_{2} \mathrm{O}_{3}$ and $\mathrm{ZrO}_{2}$ can be found in the thermodynamics manual [24], and according to the Neumann-Kopprule rule [25], the specific heat capacity of 8YSZ is calculated by the following formula for several temperature levels as listed in Table 3.

$\mathrm{C}_{\mathrm{p}-8 \mathrm{YSZ}}=0.08 \mathrm{C}_{\mathrm{p}-\mathrm{Y} 2 \mathrm{O} 3}+0.92 \mathrm{C}_{\mathrm{p}-\mathrm{ZrO} 2}$

\subsection{Modeling and verification}

According to the microstructure of the APS thermal barrier coating observed by SEM as shown in Fig. 1, it can be found that there are many pores and cracks within the coating. There're also many imperfect bonding regions between the splats, which is formed during depositing the lamellar structure and results in obvious discontinuous interfaces between the adjacent splats [20]. To quantitatively describe such discontinuous interface and analyze its effect, the discontinuity between adjacent splats is defined as the ratio of the discontinuous interface area to the total interface area for which the discontinuous interface and continuous interface are indicated by dotted lines $\left(\mathrm{S}_{\mathrm{d}}\right)$ and solid lines $\left(\mathrm{S}_{\mathrm{c}}\right)$ in Fig. 2, respectively.

$n=\frac{S_{d}}{S_{d}+S_{c}}$

Typically, the splat-interface discontinuity was calculated to be approximately $45.4 \%$ for the present samples according to the SEM photomicrographs and the statistical characteristics of the splat-interface discontinuity are shown in Table 4. These experimental measurement and other parameters as listed in Table 5 are applied to the finite element model, in which the discontinuity is particularly proposed.

Moreover, some other mathematical treatments are applied in the model as following: 1) The porosity is uniformly sprinkled into the bulk material and the thermal conductivity of the bulk material with porosity is related to the thermal conductivity of the solid 8YSZ as [26]: $k=k_{0} \times\left(1-\frac{4 \phi}{3}\right)$; 2) The continuous interface is thermally perfect conductive, while the discontinuous interface is thermally insulated without considering surface thermal radiation herein; 3) The discontinuity of each interface is identical and equals to the variable $n ; 4)$ The discontinuous interfaces distribute randomly between the adjacent splats (layers).

The calculation of the effective thermal conductivity of the model is

Table 2

The statistical characteristics of the thickness of each layer.

\begin{tabular}{|c|c|c|c|c|c|c|c|c|c|c|c|}
\hline Number & 1 & 2 & 3 & 4 & 5 & 6 & 7 & 8 & 9 & 10 & average \\
\hline Thickness $(\mu \mathrm{m})$ & 9.85 & 10.25 & 10.86 & 12.25 & 9.28 & 9.75 & 8.96 & 10.22 & 10.42 & 10.66 & 10.25 \\
\hline
\end{tabular}


Table 3

Theoretical specific heat capacity of 8YSZ

\begin{tabular}{|c|c|c|c|c|c|c|c|}
\hline Temperature $\left({ }^{\circ} \mathrm{C}\right)$ & 20 & 200 & 400 & 600 & 800 & 1000 & 1200 \\
\hline $\mathrm{C}_{\mathrm{p}-8 \mathrm{Ysz}}(\mathrm{J} /(\mathrm{g} \cdot \mathrm{K}))$ & 0.45002 & 0.54121 & 0.57813 & 0.59984 & 0.61635 & 0.63971 & 0.64461 \\
\hline
\end{tabular}
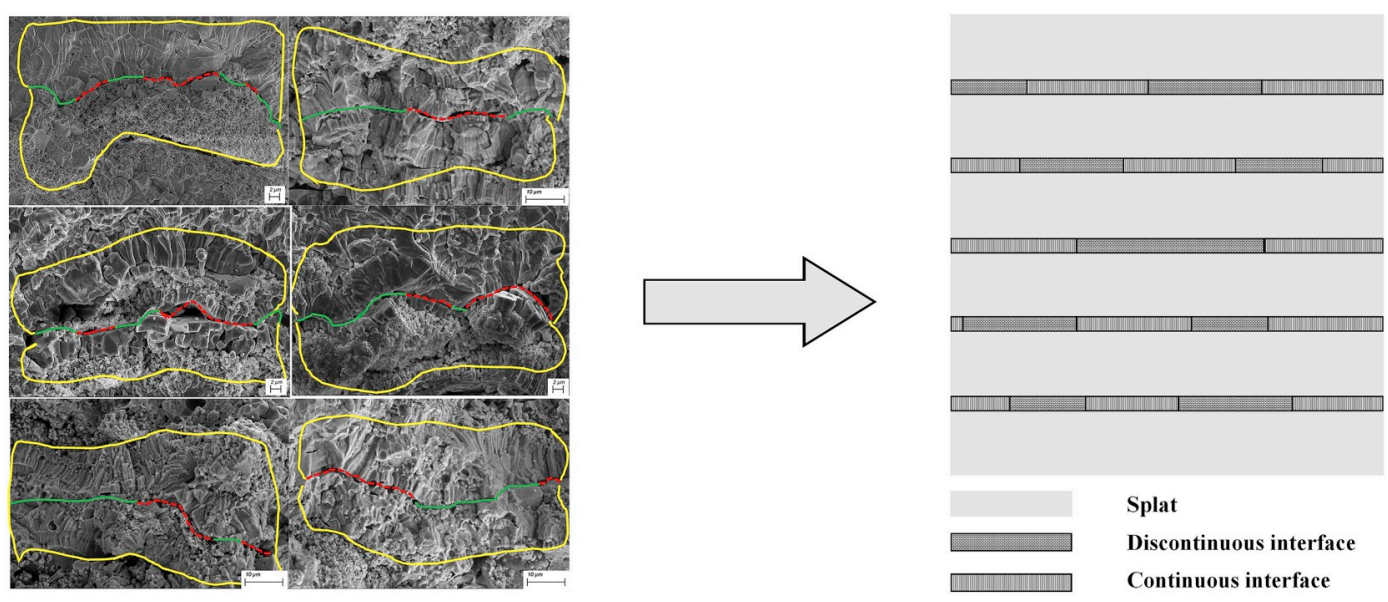

Fig. 2. The splat-interface discontinuity statistics and model simulation.

Table 4

The statistical characteristics of the splat-interface discontinuity.

\begin{tabular}{llllllll}
\hline Number & 1 & 2 & 3 & 4 & 5 & 6 & average \\
\hline $\mathrm{Sd}(\mu \mathrm{m})$ & 27.68 & 22.557 & 22.088 & 25.077 & 22.001 & 24.297 & \\
$\mathrm{Sc}(\mu \mathrm{m})$ & 32.3 & 27.793 & 28.457 & 26.843 & 23.93 & 28.753 & \\
$\mathbf{n}=\frac{\mathbf{S}_{\mathbf{d}}}{\mathbf{S}_{\mathbf{d}}+\mathbf{S}_{\mathbf{c}}}$ & $42.30 \%$ & $44.80 \%$ & $43.70 \%$ & $48.30 \%$ & $47.90 \%$ & $45.80 \%$ & $45.40 \%$ \\
\hline
\end{tabular}

Table 5

Model parameters.

\begin{tabular}{llll}
\hline Item & Thickness of each layer & Number of layers & Discontinuity \\
\hline value & 10 & 125 & $45 \%$ \\
\hline
\end{tabular}

based on the Fourier's law and thermally conductive partial differential equation.

$q=-k \nabla T$

$\rho C_{p} \frac{\partial T}{\partial t}=k \nabla^{2} T$

Where $\mathrm{q}$ is the heat flux vector, $\nabla T$ is the temperature gradient vector, $\mathrm{k}$ is thermal conductivity, $\rho$ is the material density, $C_{p}$ is the specific heat capacity and $\nabla^{2}$ is the Laplacian operator.

The effective thermal conductivity is computed with the steadystate heat conduction analysis, which is described by Eq. (6) with $\frac{\partial T}{\partial t}=0$. The given temperature boundaries are prescribed at both the top and the bottom surfaces, while the insulated boundaries at the vertical surfaces. Therefore, a certain temperature difference of $\Delta \mathrm{T}$ is established through the depth of the coating and the heat flux field is computed by the Finite Element Method, which can be used together to calculate the effective thermal conductivity with Fourier's law as

$k_{e f f}=\frac{q_{y} h}{\Delta T}$

Here, $k_{\text {eff }}$ is the effective thermal conductivity, $q_{y}$ is the total steadystate heat flux through the transverse cross-section of the model, $h$ is the thickness of the model and $\Delta T$ is the temperature difference between the top and bottom surfaces.

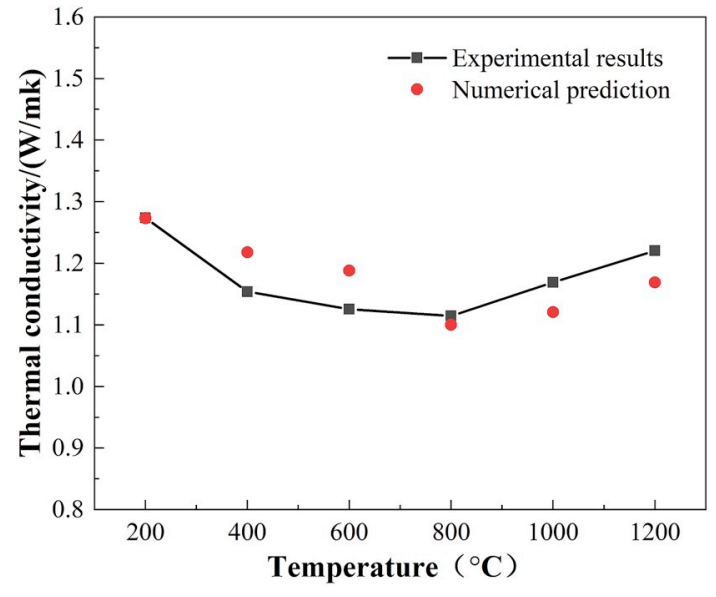

Fig. 3. Comparison of numerical results with experimental results.

The typical results of effective thermal conductivities estimated by numerical computation and measured by experiment are shown in Fig. 3, which indicate a well agreement as a whole. Although, there are small deviations in magnitudes of thermal conductivity for most points, which should be mainly due to the uncertainty of the thermal conductivity of the bulk materials that used in the finite element model.

\section{Results and discussion}

\subsection{The effects of interface discontinuity}

Based on the verified layered model APS TBCs, the effects of discontinuity on thermal conductivity were investigated. Firstly, keep the splat thickness constant, let the discontinuous interfaces randomly distribute and change the discontinuity of the interfaces, and the models with different degree of interface discontinuity are shown in Fig. 4. The effective thermal conductivity of the coating was computed at different levels of temperature and the results are shown in Fig. 5, in which it can be found that the effective thermal conductivity would decrease with increasing the interface discontinuity if the layer thickness is kept constant. The results can also partially explain why the 

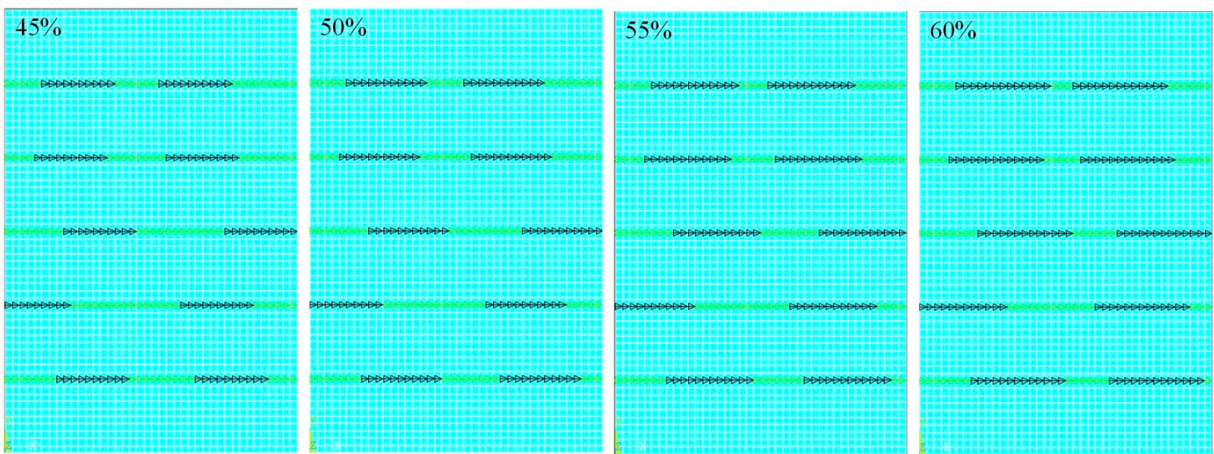

Fig. 4. Finite element models with different splat-interface discontinuity.

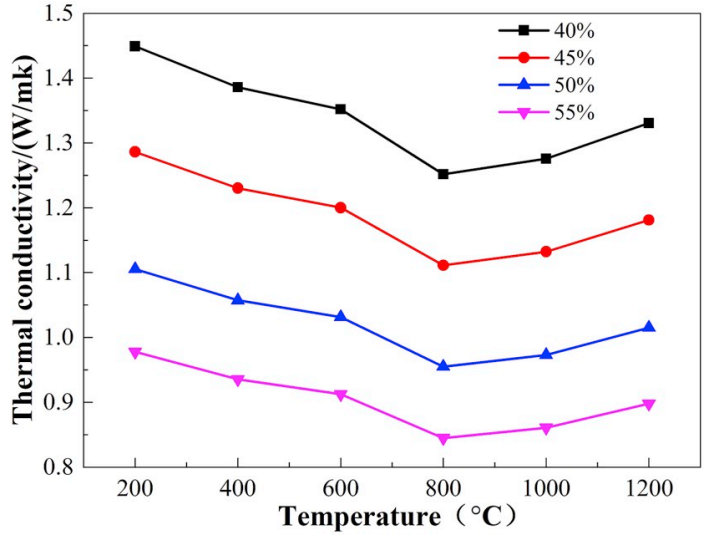

Fig. 5. Effective thermal conductivity of TBCs with different splat-interface discontinuity.

thermal conductivity of the thermal barrier coating will increase after high temperature heat treatment [27]. Since the thermal barrier coating is heat-treated at a high temperature, the discontinuous interface between the layers is fused and converted into a continuous interface [28], which means that the degree of interface discontinuity of the coating is reduced and therefore the thermal conductivity increased.

Then on, keep the discontinuity and the total thickness of the sample fixed, change the thickness of each single layer, which is actually the vertical distances between the adjacent discontinuous interfaces as shown in Fig. 6.

It is indicated in Fig. 7 that the effective thermal conductivity of the coating would increase with increasing the layer thickness, as which means that density of the longitudinal distribution of discontinuous interfaces decrease. That is to say, the coating changes into a more continuous media if the thickness of each layer is increased.

\subsection{The effect of the distribution of discontinuous interfaces}

The previously discussed on the effects of discontinuity and layer thickness are conducted for the cases of random distribution of

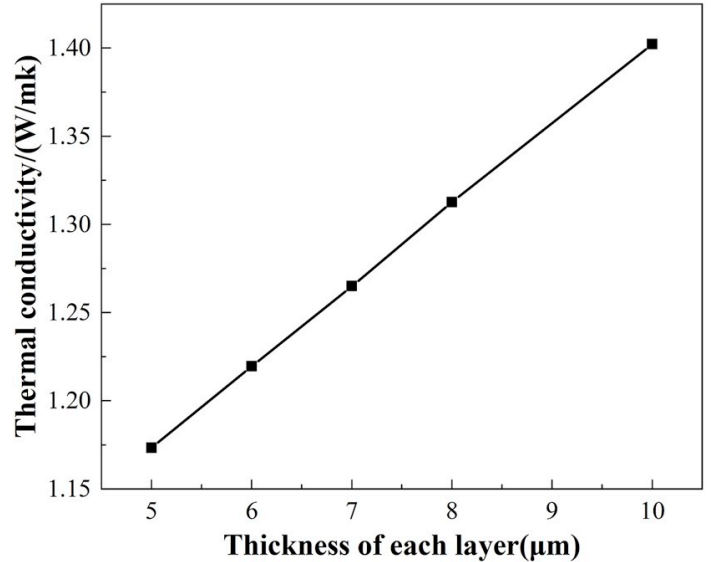

Fig. 7. Effect of thickness of a single layer on the effective thermal conductivity of the coating.

discontinuous interfaces. As a matter of fact, the distribution of discontinuous interfaces is controllable in some extent $[8,29,30]$, which means that the thermal performance of the coating could be further tailored. Of course, the effect of the distribution of the discontinuous interfaces would be revealed herein to find out the probably existence of the most optimization. This theoretical investigation is carried through by keeping the discontinuity and the layer thickness fixed. Without loss of generality, a tri-layer model with two interfaces is adopted in this analysis, in which only one section of discontinuous interface is taken into account for each interface as shown in Fig. 8(a).

The optimal solution to the relative location of the two discontinuous interfaces is derived with the parameters of layer thickness $\mathrm{d}$, total width a, and length of discontinuity $\mathrm{n}$ is variably distributed. An ideal crack originated at $x_{1}$ is assumed to attribute for the section of the discontinuous interface between the top layer and the middle layer, and an ideal crack originated at $x_{2}$ for that between the middle layer and the bottom layer. Therefore, the heat flow from top surface to bottom surface has three heat transfer paths $\mathrm{q}_{1}, \mathrm{q}_{2}, \mathrm{q}_{3}$ in parallel. The total heat flow could be written as following if only the shortest heat conduction
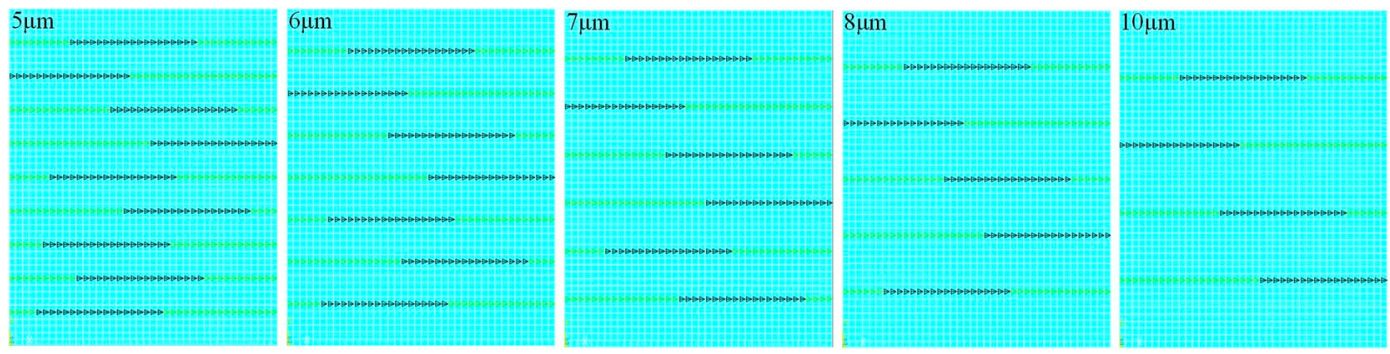

Fig. 6. Finite element models with different distances between discontinuous interfaces. 


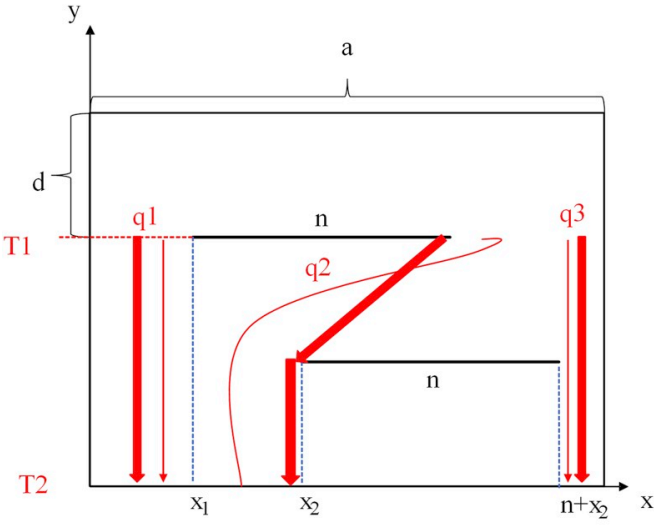

(a)

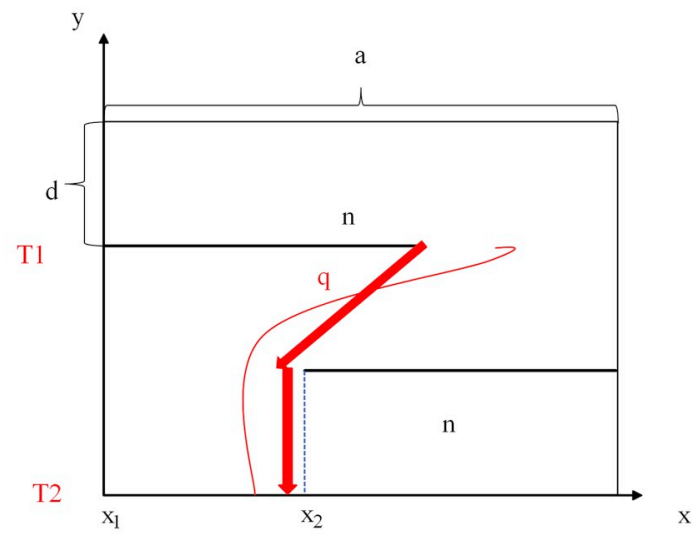

(b)

Fig. 8. Sketch of the (a) general and (b) optimal distribution of discontinuous interfaces.

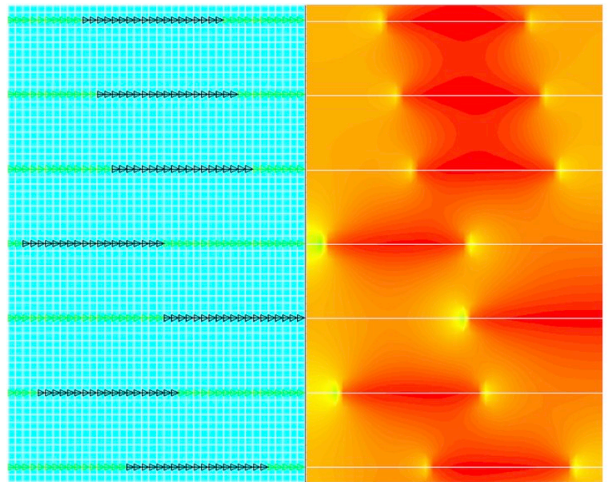

(a) Random distribution model

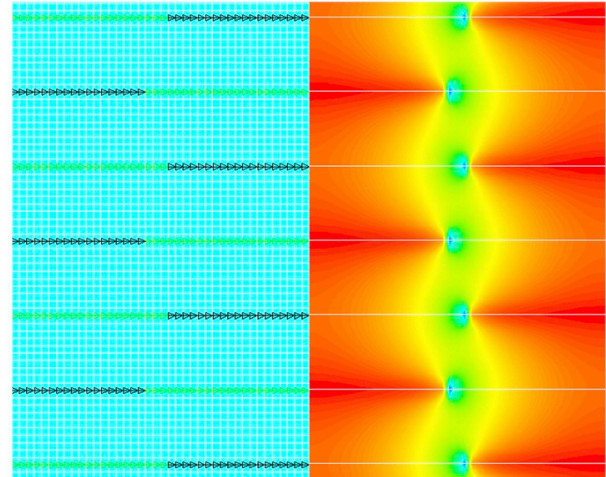

(b) Overlapping distribution model

\begin{tabular}{rlllllllll}
\hline$-.112 \mathbb{E}+07$ & -996170 & -874038 & -751906 & -629774 & -507642 & -385510 & -263378 & -141246 & -1667.08
\end{tabular}

Fig. 9. Heat flux field for the cases of random and overlapping discontinuous interfaces.

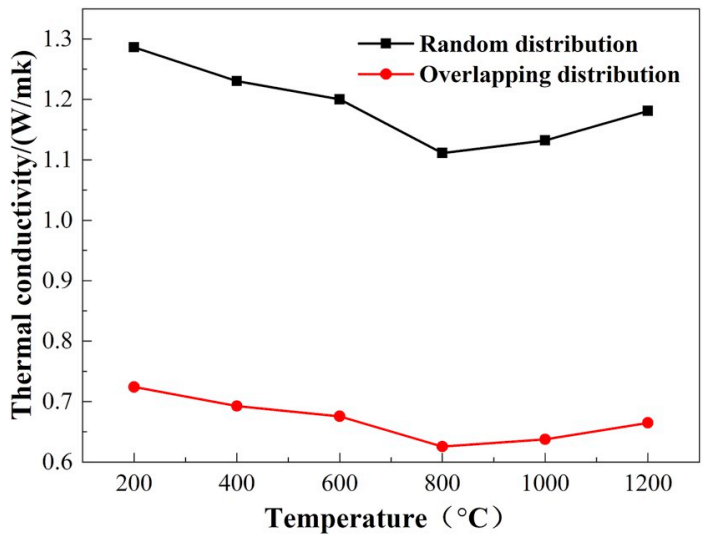

Fig. 10. Effective thermal conductivity for the cases of random and overlapping discontinuous interfaces.

path is considered.

$$
\begin{aligned}
Q_{\text {total }}= & Q_{1}+Q_{2}+Q_{3}=\int_{0}^{x_{1}} k \frac{\Delta T}{2 d}+\int_{x_{1}}^{x_{2}} k \frac{\Delta T}{\sqrt{d^{2}+\left(n+x_{1}-x_{2}\right)^{2}}+d} \\
& +\int_{n+x_{2}}^{a} k \frac{\Delta T}{2 d}
\end{aligned}
$$

Which is,

$$
Q_{\text {total }}=k \Delta T\left(\frac{x_{1}}{2 d}+\frac{x_{2}-x_{1}}{\sqrt{d^{2}+\left(n+x_{1}-x_{2}\right)^{2}}+d}+\frac{a-x_{2}-n}{2 d}\right) \sim f\left(x_{1}, x_{2}\right)
$$

As we can understand that, the less the total heat flow, the lower the effective thermal conductivity. At the same time, to minimize the quantity $Q_{\text {total }}$ is to minimize the function $\mathrm{f}\left(\mathrm{x}_{1}, \mathrm{x}_{2}\right)$, that is

$$
\begin{gathered}
f\left(x_{2}-x_{1}\right)=f(y)=k \Delta T\left(\frac{a-n-y}{2 d}+\frac{y}{\sqrt{d^{2}+(n-y)^{2}}+d}\right)= \\
k \Delta T\left[\frac{a-n}{2 d}+y\left(\frac{1}{\sqrt{d^{2}+(n-y)^{2}}+d}-\frac{1}{2 d}\right)\right]
\end{gathered}
$$

Immediately, the minimum value is obtained when $y=x_{2}-x_{1}=a-n$ with $\mathrm{x}_{2}=\mathrm{a}-\mathrm{n}$ and $\mathrm{x}_{1}=0$, which is the configuration of overlapping discontinuous interfaces as shown in Fig. 8(b).

Therefore, it is revealed by the present solution that the configure of overlapping discontinuous interfaces has the lowest effective thermal conductivity and should be regarded as the most optimized option, which is further verified by finite element analysis, which involves a multilayer structure for generality. Typically, the contours of heat flux field in the sample subjected to specified temperature boundaries are shown in Fig. 9, wherein Fig. 9(a) and (b) represents the cases of random distribution of discontinuous interfaces and overlapping 

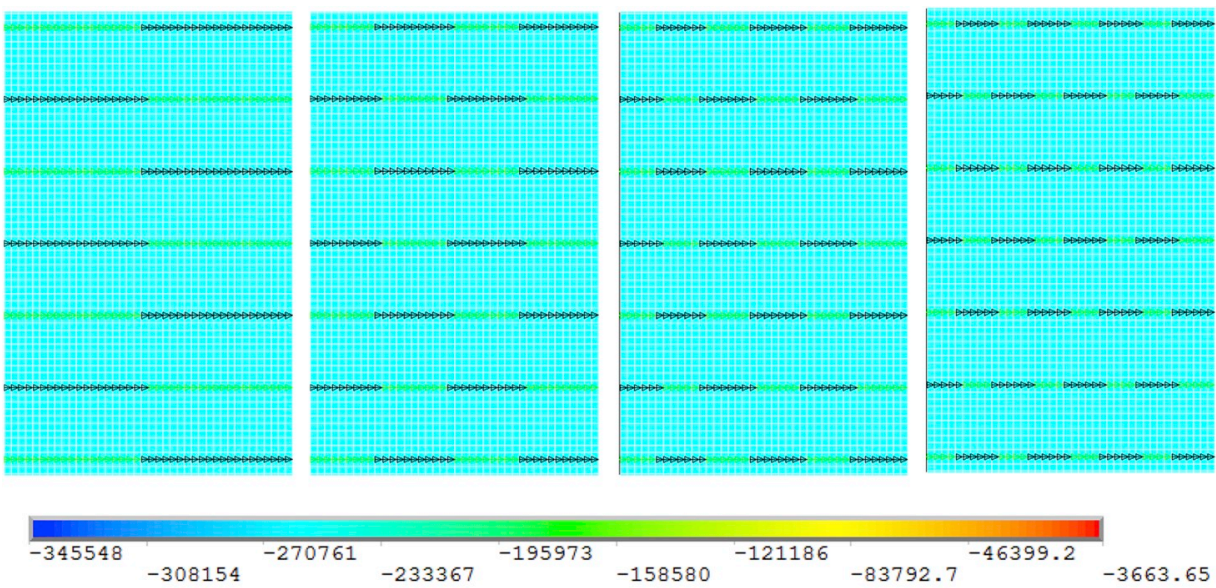

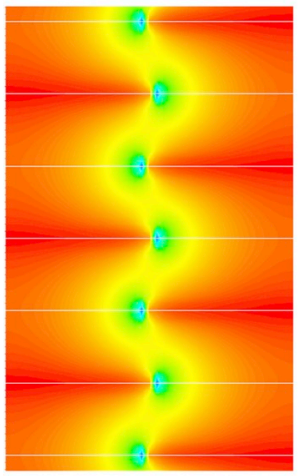

(a) $1+1$

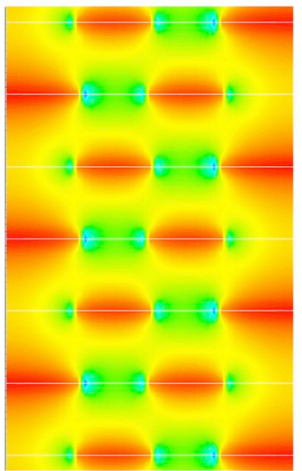

(b) $2+2$

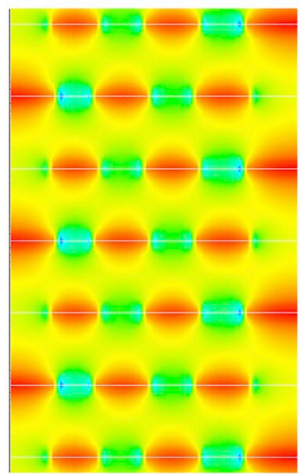

(c) $3+3$

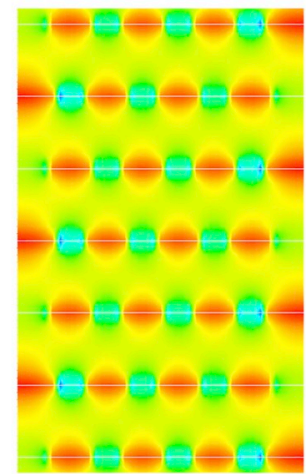

(d) $4+4$

Fig. 11. The heat flow density map of different distribution model.

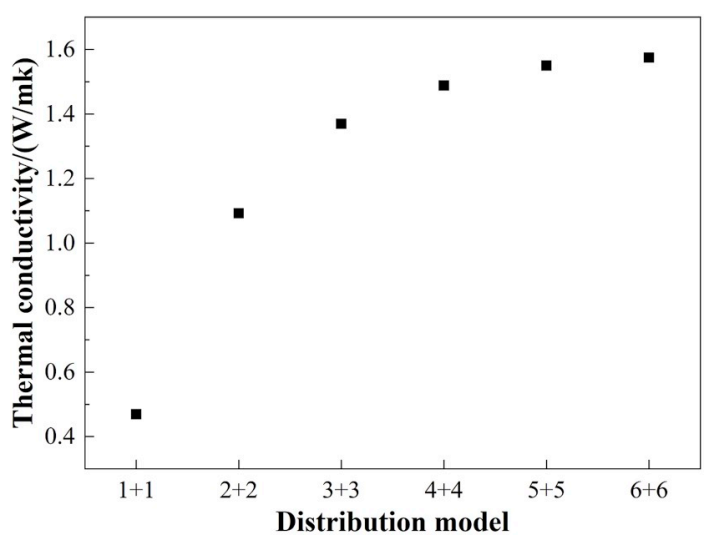

Fig. 12. Effect of distribution of discontinuous interfaces on thermal conductivity.

distribution of discontinuous interfaces, respectively. and the effective thermal conductivity of the cases of random and overlapping distribution of discontinuous interfaces is computed and compared shown in Fig. 10.

It is shown that the overlapping distribution of discontinuous interfaces would be the optimal solution to minimize the effective thermal conductivity of the multilayer coating when there is only one discontinuous interface between two adjacent layers. It is also indicated that the distribution of overlapping discontinuous interfaces would maximize the heat transfer path of the heat flow, which decrease the effective thermal conductivity. The effect of the dispersity of the interface discontinuity is further investigated with dividing the discontinuous interface into different numbers of section while keep the interface discontinuity fixed, which means the total length of the discontinuous interfaces is identical. The optimized case of overlapping discontinuous interfaces is always adopted in this model as shown in the upper part of Fig. 11 and the typical contours of heat flux field are shown in the lower part of Fig. 11. Further, the effect of the dispersion degree of the discontinuous interfaces on the effective thermal conductivity of the coating is shown in Fig. 12.

It is shown that the effective thermal conductivity would increase with increasing the dispersity of the interface discontinuity, which means that the thermal barrier ability of the interface discontinuity would decrease with its degree of dispersion. This is due to the fact that the increase of dispersion of the interface discontinuity provides more outlets and shortcuts for the heat flow to pass through as shown in Fig. 12, which shorten the heat transfer path and therefore enhance the apparent thermal conductivity. However, the effective thermal conductivity would converge into some steady value with increasing the dispersity of the interface discontinuity to some extremity. This means that the shortest heat flow heat transfer path has been realized for the case of specified degree of interface discontinuity that is fully dispersed.

\section{Conclusions}

The effects of interface discontinuity of the APS TBC on its effective thermal conductivity are investigated and it is found that the effective thermal conductivity of the coating could be optimized by designing the 
topological features of the discontinuous interfaces. The detailed results could be summarized as following.

(1) The effective thermal conductivity of the coating would be reduced with increasing the interface discontinuity when the layer thickness is fixed.

(2) The effective thermal conductivity of the coating would increase with increasing the layer thickness of the splats when the interface discontinuity is fixed.

(3) The overlapping distribution of the discontinuous interfaces is found to be the optimized status for minimizing the effective thermal conductivity of the coating.

(4) The effective thermal conductivity of the coating would increase with increasing the dispersion of the discontinuous interfaces, although the thermal conductivity would approach to some steady value when the dispersion is large enough.

\section{Declaration of competing interests}

The authors declare that they have no known competing financial interests or personal relationships that could have appeared to influence the work reported in this paper.

\section{Acknowledgments}

This work was supported by the Strategic Priority Research Program of Chinese Academy of Sciences (Grant No. XDA17030100 and XDA17030200) and the National Natural Science Foundation of China under grant No. 11572327, U1537212 and No. 51471019.

\section{References}

[1] R.L. Jones, Thermal barrier coatings, in: K.H. Stern (Ed.), Metallurgical and Ceramic Protective Coatings, Springer Netherlands, Dordrecht, 1996, pp. 194-235.

[2] A.G. Evans, D.R. Mumm, J.W. Hutchinson, G.H. Meier, F.S. Pettit, Mechanisms controlling the durability of thermal barrier coatings, Prog. Mater. Sci. 46 (5) (2001) 505-553.

[3] N.P. Padture, M. Gell, E.H. Jordan, Thermal barrier coatings for gas-turbine engine applications, Science 296 (5566) (2002) 280.

[4] A. Rabiei, A.G. Evans, Failure mechanisms associated with the thermally grown oxide in plasma-sprayed thermal barrier coatings, Acta Mater. 48 (15) (2000) 3963-3976.

[5] K.W. Schlichting, N.P. Padture, E.H. Jordan, M. Gell, Failure modes in plasmasprayed thermal barrier coatings, Mater. Sci. Eng. A 342 (1) (2003) 120-130.

[6] W. Chi, S. Sampath, H. Wang, Microstructure-thermal conductivity relationships for plasma-sprayed yttria-stabilized zirconia coatings, J. Am. Ceram. Soc. 91 (8) (2008) 2636-2645.

[7] N. Markocsan, P. Nylén, J. Wigren, X.H. Li, Low thermal conductivity coatings for gas turbine applications, J. Therm. Spray Technol. 16 (4) (2007) 498-505.

[8] A. Jadhav, N. Padture, E. Jordan, M. Gell, P. Miranzo, E. Fullerjr, Low-thermalconductivity plasma-sprayed thermal barrier coatings with engineered microstructures, Acta Mater. 54 (12) (2006) 3343-3349.
[9] C. Zhang, J. Fei, L. Guo, J. Yu, B. Zhang, Z. Yan, F. Ye, Thermal cycling and hot corrosion behavior of a novel LaPO4/YSZ double-ceramic-layer thermal barrier coating, Ceram. Int. 44 (8) (2018) 8818-8826.

[10] L. Guo, Z. Yan, Z. Li, J. Yu, Q. Wang, M. Li, F. Ye, GdPO4 as a novel candidate for thermal barrier coating applications at elevated temperatures, Surf. Coat. Technol. 349 (2018) 400-406.

[11] Q. Wang, L. Guo, Z. Yan, F. Ye, Phase composition, thermal conductivity, and toughness of TiO2-doped, Er2O3-stabilized $\mathrm{ZrO} 2$ for thermal barrier coating applications, Coatings 8 (7) (2018).

[12] L. Guo, C. Zhang, L. Xu, M. Li, Q. Wang, F. Ye, C. Dan, V. Ji, Effects of TiO2 doping on the defect chemistry and thermo-physical properties of $\mathrm{Yb} 2 \mathrm{O} 3$ stabilized $\mathrm{ZrO} 2$, J. Eur. Ceram. Soc. 37 (13) (2017) 4163-4169.

[13] F. Wu, P. Wu, L. Chen, J. Feng, Structure and thermal properties of Al2O3-doped Gd3TaO7 as potential thermal barrier coating, J. Eur. Ceram. Soc. 39 (2019) 2210-2214.

[14] X.Q. Jin, C.Y. Zhao, Numerical investigation on the effective thermal conductivity of plasma sprayed zirconia coatings, Ceram. Int. 41 (10) (2015) 14915-14923.

[15] S. Wei, W. Fu-chi, F. Qun-Bo, M. Zhuang, Effects of defects on the effective thermal conductivity of thermal barrier coatings, Appl. Math. Model. 36 (5) (2012) 1995-2002.

[16] I. Sevostianov, M. Kachanov, Plasma-sprayed ceramic coatings: anisotropic elastic and conductive properties in relation to the microstructure; cross-property correlations, Mater. Sci. Eng. A 297 (2001) 235-243.

[17] T. Nakamura, G. Qian, C.C. Berndt, Effects of pores on mechanical properties of plasma-sprayed ceramic coatings, J. Am. Ceram. Soc. 83 (2000) 578-584.

[18] Y. Wang, J. Hua, Z. Liu, Y. Zeng, Y. Zhou, H. Wang, Melting index characterization and thermal conductivity model of plasma sprayed YSZ coatings, J. Eur. Ceram. Soc. 32 (14) (2012) 3701-3707.

[19] A. Kulkarni, Z. Wang, T. Nakamura, S. Sampath, A. Goland, H. Herman, J. Allen, J. Ilavsky, G. Long, J. Frahm, R.W. Steinbrech, Comprehensive microstructural characterization and predictive property modeling of plasma-sprayed zirconia coatings, Acta Mater. 51 (9) (2003) 2457-2475.

[20] Z. Wang, A. Kulkarni, S. Deshpande, T. Nakamura, H. Herman, Effects of pores and interfaces on effective properties of plasma sprayed zirconia coatings, Acta Mater. 51 (2003) 5319-5334.

[21] I.O. Golosnoy, S.A. Tsipas, T.W. Clyne, An analytical model for simulation of heat flow in plasma-sprayed thermal barrier coatings, J. Therm. Spray Technol. 14 (2) (2005) 205-214.

[22] L. Lu, F.-C. Wang, Z. Ma, Q.-B. Fan, Anisotropic effect of splat interface on thermal conductivity of plasma sprayed YSZ coating, Surf. Coat. Technol. 235 (2013) 596-602.

[23] K.D. Maglic, A. Cezairliyan, V.E. Peletsky, Compendium of Thermophysical Property Measurement Methods vol. 1, survey of measurement techniques, 1984.

[24] Y.J. Liang, Y.C. Che, X.X. Liu, N.J. Li, Manual of Practical Inorganic Matter Thermodynamics, Northeastern University Press, Shenyang, 1993.

[25] J. Leitner, P. Chuchvalec, D. Sedmidubský, A. Strejc, P. Abrman, Estimation of heat capacities of solid mixed oxides, Thermochim. Acta 395 (1-2) (2002) 27-46.

[26] P.G. Klemens, Thermal conductivity of inhomogeneous media, High. Temp. - High. Press. 23 (3) (1991) 241-248.

[27] J. Yu, H. Zhao, S. Tao, X. Zhou, C. Ding, Thermal conductivity of plasma sprayed Sm2Zr2O7 coatings, J. Eur. Ceram. Soc. 30 (3) (2010) 799-804.

[28] J. Yu, H. Zhao, X. Zhou, S. Tao, C. Ding, Effect of thermal aging on microstructure and mechanical properties of plasma-sprayed samarium zirconate coatings, J. Therm. Spray Technol. 20 (5) (2011) 1056-1062.

[29] T. Liu, S.-L. Zhang, X.-T. Luo, G.-J. Yang, C.-X. Li, C.-J. Li, High heat insulating thermal barrier coating designed with large two-dimensional inter-lamellar pores, J. Therm. Spray Technol. 25 (1-2) (2015) 222-230.

[30] T. Liu, X. Chen, G.-J. Yang, C.-J. Li, Properties evolution of plasma-sprayed $\mathrm{La}_{2} \mathrm{Zr}_{2} \mathrm{O}_{7}$ coating induced by pore structure evolution during thermal exposure, Ceram. Int. 42 (14) (2016) 15485-15492. 